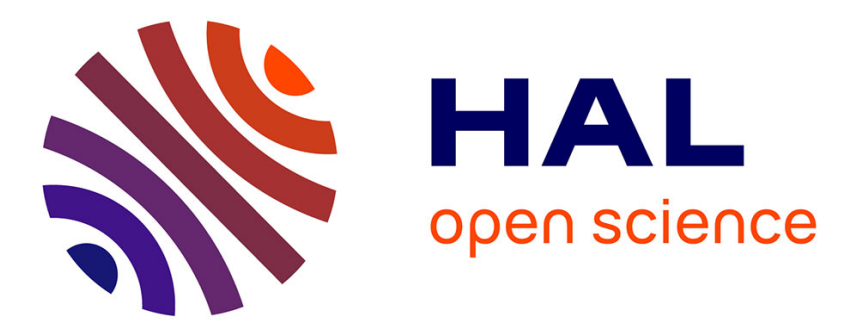

\title{
Vortex-Nucleating Zeeman Resonance in Axisymmetric Rotating Bose-Einstein Condensates
}

\author{
G. Reinisch
}

\section{To cite this version:}

G. Reinisch. Vortex-Nucleating Zeeman Resonance in Axisymmetric Rotating Bose-Einstein Condensates. Physical Review Letters, 2007, 99, pp.120402. 10.1103/PhysRevLett.99.120402 . hal00388157

\section{HAL Id: hal-00388157 \\ https://hal.science/hal-00388157}

Submitted on 13 Jan 2022

HAL is a multi-disciplinary open access archive for the deposit and dissemination of scientific research documents, whether they are published or not. The documents may come from teaching and research institutions in France or abroad, or from public or private research centers.
L'archive ouverte pluridisciplinaire HAL, est destinée au dépôt et à la diffusion de documents scientifiques de niveau recherche, publiés ou non, émanant des établissements d'enseignement et de recherche français ou étrangers, des laboratoires publics ou privés. 


\title{
Vortex-Nucleating Zeeman Resonance in Axisymmetric Rotating Bose-Einstein Condensates
}

\author{
Gilbert Reinisch \\ Département Cassiopée, Observatoire de la Côte d'Azur, BP 4229, 06304 Nice Cédex 4, France
}

(Received 26 January 2007; published 18 September 2007)

\begin{abstract}
By use of the Larmor equivalence between uniform rotation and a magnetic field, we consider in the strong-interaction Thomas-Fermi regime the single centered vortex as the first Zeeman-like excited state of the axisymmetric rotating Bose-Einstein condensate. This yields a resonant-drive nucleation mechanism whose threshold is in quite good agreement with ENS, MIT, and JILA experimental results.
\end{abstract}

DOI: 10.1103/PhysRevLett.99.120402

PACS numbers: 03.75.Lm

I. Introduction. - The Bose-Einstein condensate (BEC), first observed in 1995 in a remarkable series of experiments on atomic vapors [1-3], is an outstanding milestone in experimental and theoretical research on quantum statistical mechanics [4,5], raising in particular the question of whether a BEC state exhibits a mesoscopic quantum analogue of the macroscopic vortices in superfluids [6], and what its experimental signature should be. And indeed, the prediction of the steady states of a confined, rotating dilute Bose gas by calculating the macroscopically occupied condensate wave function that minimizes the total energy of the gas at fixed angular velocity [7] was soon followed by the observation of vortex formation [8-13] and vortex lattices [14] in a so-called stirred (i.e., rotating) BEC.

Vortex nucleation in a dilute gaseous rotating BEC is a recurrent and difficult problem [15]. More than half a decade after the pioneering experiments done by the Boulder-JILA [8,9], the Paris-ENS [10-12], and the MIT $[13,14]$ groups, a few controversial points remain that were quickly raised by theoretical and/or numerical investigations [16-20]: (i) the vortex state is not a global energy minimum [19-21], (ii) the dynamic instability threshold due to nonlinear excitation of surface waves is consistent with the ENS results [17], but not with the MIT [13] and JILA [9] ones, and (iii) predicted hysteresis phenomena in the vortex nucleation due to the metastabilization of the vortexless condensate $[19,20]$ have not been observed [12].

One possible reason for these discrepancies might be that the BEC rotation is not properly taken into account. In the present Letter, we use a standard Zeeman approach based on the quantum mechanical equivalence between uniform rotation and magnetic vector potential in order to propose a theory that just fits with all above ENS, MIT, and JILA experimental results. This approach has widely been used in the rapidly rotating quasilinear case where the weak nonlinear interactions between atoms can be neglected as a result of the almost total screening of the harmonic trapping potential by the centrifugal one. Then one basically recovers the well-known linear Landau picture [22-25]. In contrast to this linear approach, we address in the present Letter the highly nonlinear strong- interaction case which is well described by the ThomasFermi (TF) approximation $[4,5,26]$. The corresponding Zeeman-Thomas-Fermi (ZTF) investigation of a rotating $\mathrm{BEC}$ is new.

II. Zeeman description of a rotating BEC. - The wellknown quantum description of a nonrelativistic spinless particle with charge $e$ and mass $M$ confined in the external potential $V$ and moving in the uniform magnetic field $\overrightarrow{\mathcal{B}}=$ $\nabla \times \overrightarrow{\mathcal{A}}$ defined by the Coulomb-gauge vector potential $\overrightarrow{\mathcal{A}}=\frac{1}{2} \overrightarrow{\mathcal{B}} \times \overrightarrow{\mathbf{r}}$ yields the Hamiltonian

$$
\mathcal{H}=\frac{1}{2 M}[-i \hbar \nabla-\overrightarrow{\mathbf{p}}]^{2}+V=H-\overrightarrow{\mathbf{\Omega}} \cdot \overrightarrow{\mathbf{L}}+\frac{p^{2}}{2 M},
$$

where $\overrightarrow{\mathbf{p}}=(e / c) \overrightarrow{\mathcal{A}}$ and $\overrightarrow{\mathbf{L}}$ is the quantum mechanical angular momentum operator per particle, while $H$ is the corresponding Hamiltonian in the absence of any magnetic field. The Larmor vector $\overrightarrow{\boldsymbol{\Omega}}=(e / 2 M c) \overrightarrow{\mathcal{B}}$ yields $\overrightarrow{\mathbf{p}}=$ $M \overrightarrow{\boldsymbol{\Omega}} \times \overrightarrow{\mathbf{r}}$, which has the transparent physical meaning of a uniform rotation field defined by the velocity streamlines $\overrightarrow{\mathbf{v}}=\overrightarrow{\mathbf{p}} / M=\overrightarrow{\boldsymbol{\Omega}} \times \overrightarrow{\mathbf{r}}$. Note that Eq. (1) can be rewritten as

$$
H-\overrightarrow{\boldsymbol{\Omega}} \cdot \overrightarrow{\mathbf{L}}=\frac{1}{2 M}[-i \hbar \nabla-\overrightarrow{\mathbf{p}}]^{2}+V-\frac{p^{2}}{2 M},
$$

which provides the description in the corotating frame of the quantum particle driven by the Coriolis force and trapped in that "rotating" potential $V_{\text {rot }}=V-p^{2} / 2 M$. Equivalently, the right-hand side of Eq. (1) defines the well-known Zeeman Hamiltonian $\mathcal{H}$. The only departure from the standard Zeeman description consists in retaining the $p^{2} / 2 M$ diamagnetic quadratic term because it is of the same order of magnitude as the $-\overrightarrow{\boldsymbol{\Omega}} \cdot \overrightarrow{\mathbf{L}}$ standard paramagnetic Zeeman coupling.

The eigenvalue of the nonlinear Zeeman Hamiltonian $\mathcal{H}$ defined by Eq. (1) and including the Gross-Pitaevskii (GP) nonlinear interaction term $V_{\mathrm{GP}}=\kappa|\Psi(\mathbf{r})|^{2}$ (where the coupling constant $\kappa=4 \pi N \hbar^{2} a_{s} / M$ is defined by the condensate number $N$ and the positive scattering length $a_{s}$ ) becomes the chemical potential $\mu$, according to the following stationary GP differential equation: 


$$
\mathcal{H} \Psi=\left[H+V_{\mathrm{GP}}-\overrightarrow{\boldsymbol{\Omega}} \cdot \overrightarrow{\mathbf{L}}+\frac{p^{2}}{2 M}\right] \Psi=\mu \Psi .
$$

Quantum averaging Eq. (3) (hence the brackets) and taking into account $\left\langle V_{\mathrm{GP}}\right\rangle=2 E_{\text {int }}$, where $E_{\text {int }}$ is the average interaction energy per particle [4,5,26], yields $E+E_{\text {int }}=\mu$, where $E=\langle H\rangle+E_{\text {int }}-\langle\overrightarrow{\boldsymbol{\Omega}} \cdot \overrightarrow{\mathbf{L}}\rangle+\left\langle p^{2} / 2 M\right\rangle$ is the total condensate energy per particle in the rotation field $\overrightarrow{\boldsymbol{\Omega}}$. Eliminating $E_{\text {int }}$, we obtain

$$
E=\frac{1}{2}\left[\mu+\langle H\rangle-\langle\overrightarrow{\boldsymbol{\Omega}} \cdot \overrightarrow{\mathbf{L}}\rangle+\left\langle\frac{p^{2}}{2 M}\right\rangle\right] .
$$

Let us now assume that the BEC is confined in the transverse harmonic potential $V=\frac{1}{2} M r^{2} \omega_{\perp}^{2}$ and that we have the cylindrical symmetry $r, \theta, z$ where $\overrightarrow{\mathbf{L}}=\left\{0,0, L_{z}\right\}$ and $\overrightarrow{\boldsymbol{\Omega}}=\{0,0, \Omega\}$, thus yielding $p^{2} / 2 M=\frac{1}{2} M r^{2} \Omega^{2}$. The case $L_{z}=0$ defines the vortexless ground state while $L_{z}=\hbar$ defines the first centered vortex state (remember that if it were not centered, $L_{z}$ could be a fractional number of $\hbar$ [7]). Defining both the effective confining potential $V_{\text {eff }}=$ $\frac{1}{2} M r^{2} \omega_{\text {eff }}^{2}$, where $\omega_{\text {eff }}=\sqrt{\omega_{\perp}^{2}+\Omega^{2}}$, and the corresponding effective diamagnetic Hamiltonian $H_{\text {eff }}=H+$ $p^{2} / 2 M=[-i \hbar \nabla]^{2} / 2 M+V_{\text {eff }}$, we, respectively, obtain for Eqs. (3) and (4):

$$
\begin{aligned}
{\left[H_{\mathrm{eff}}+V_{\mathrm{GP}}\right] \Psi_{\mathrm{eff}} } & =\mu_{\mathrm{eff}} \Psi_{\mathrm{eff}} ; \\
E & =\frac{1}{2}\left[\mu_{\mathrm{eff}}-2 \Omega L_{z}+\left\langle H_{\mathrm{eff}}\right\rangle\right] \\
& =E_{\mathrm{eff}}-\Omega L_{z},
\end{aligned}
$$

where $\mu_{\text {eff }}=\mu+\Omega L_{z}$ and $\left\langle H_{\text {eff }}\right\rangle=\left\langle\Psi_{\text {eff }}\left|H_{\text {eff }}\right| \Psi_{\text {eff }}\right\rangle$. As a consequence, $E_{\text {eff }}=\frac{1}{2}\left[\mu_{\text {eff }}+\left\langle H_{\text {eff }}\right\rangle\right]$ is the energy of the system in the fixed (nonrotating) frame and Eq. (5a) is its corresponding stationary GP equation.

III. Zeeman-Thomas-Fermi approximation.-We now wish to investigate the strong TF particle-particle interaction case $\Omega<\omega_{\perp}$. The quantum kinetic energy related to the Laplacian term in the Schrödinger Hamiltonian $H$ can then be neglected [4,5,17,26]. Assume $\Psi_{m}(r, \theta)=$ $a_{m}(r) e^{i m \theta}$ and define the following dimensionless variables:

$$
\begin{gathered}
\alpha_{m}=\left(\frac{\hbar \omega_{\mathrm{eff}}}{2 \mu_{\mathrm{eff}}^{m}}\right)^{2}, \quad X=r \frac{\sqrt{2 M \mu_{\mathrm{eff}}^{m}}}{\hbar}, \\
u_{m}(X)=\sqrt{\frac{\kappa}{\mu_{\mathrm{eff}}^{m}}} a_{m}[r(X)] .
\end{gathered}
$$

Then Eq. (5a) becomes

$$
\begin{aligned}
\ddot{u}_{m}(X)+ & \frac{1}{X} \dot{u}_{m}(X) \\
& +\left[1-\alpha_{m} X^{2}-\frac{m^{2}}{X^{2}}-u_{m}^{2}(X)\right] u_{m}(X)=0,
\end{aligned}
$$

where the overdot denotes $d / d X$. Given the vortex quantum circulation integer number $m$, Eq. (7) only depends on the parameter $\alpha_{m}$ which is determined by the normalization constraint:

$$
\begin{aligned}
\int|\Psi|^{2} d \mathbf{r} & =2 \pi \Delta_{z} \int a_{m}(r)^{2} r d r=1 \rightarrow \int_{0}^{\infty} u_{m}^{2}(X) X d X \\
& =\frac{4 N a_{s}}{\Delta_{z}}=\mathcal{N},
\end{aligned}
$$

where $\Delta_{z}$ is the overall BEC length in the $(z)$ axial direction. Note that $\mathcal{N}=4 \sqrt{\pi / 2} \gamma \sim 5 \gamma$, where $\gamma$ is a dimensionless parameter that measures the importance of interatomic collisions relative to the confinement of the trap $[7,20]$.

The system Eqs. (7) and (8) self-consistently defines the nonlinear eigenstate $u_{m}(X)$ of angular momentum $L_{z}=m \hbar$ related to the chemical potential $\mu_{\mathrm{eff}}^{m}=\hbar \omega_{\mathrm{eff}} /\left(2 \sqrt{\alpha_{m}}\right) \propto$ $\sqrt{\omega_{\perp}^{2}+\Omega^{2}}$ [cf. Eqs. (6)]. It will be analytically solved by use of the semiclassical TF approximation which is quite relevant in the case of a strong-interaction rotating BEC $[4,5,26]$. Indeed the number of particles $N$ in the condensate is very large and the interatomic repulsion is strong (the above interatomic collision parameter $\gamma \sim 6$ while $\mathcal{N} \sim 30$ ). Therefore the Laplacian [namely the two first terms of the left-hand side of Eq. (7)] which defines the quantum kinetic energy can be neglected and Eqs. (7) and (8), respectively, yield the TF solution $u_{m}^{2}(X) \sim 1-$ $\alpha_{m} X^{2}-\left(m^{2} / X^{2}\right)$, together with the norm condition

$$
\begin{aligned}
\frac{1}{\alpha_{m}} \int_{t_{-}}^{t_{+}}\left[1-t-\frac{m^{2} \alpha_{m}}{t}\right] d t & =\frac{1}{2 \alpha_{m}}+m^{2}\left[\ln \left(m^{2} \alpha_{m}\right)-1\right] \\
& =2 \mathcal{N},
\end{aligned}
$$

in the terms of $t=\alpha_{m} X^{2}$, where $t_{-}=m^{2} \alpha_{m}$ (respectively, $t_{+}=1-m^{2} \alpha_{m}$ ) is the lower (respectively, upper) bound for $t$ that yields positive values of $u_{m}^{2}$ in the TF limit $\alpha_{m} \ll$ 1. The problem is solved: Given the experimental parameters which define $\mathcal{N}$ and the BEC rotation frequency $\Omega$, the implicit solution $\alpha_{m}$ of Eq. (9) defines the corresponding chemical potential $\mu_{\text {eff }}^{m}=\hbar \sqrt{\omega_{\perp}^{2}+\Omega^{2}} /\left(2 \sqrt{\alpha_{m}}\right)$, as well as the integration bounds $t_{ \pm}$. Hence the analytical expression of the quantum expectation value of the effective diamagnetic TF Hamiltonian $H_{\text {eff }}=\mu_{\text {eff }}^{m} \alpha_{m} X^{2}+$ $\left(m^{2} \mu_{\text {eff }}^{m} / X^{2}\right)$ which now only includes the effective confining potential energy $V_{\text {eff }}=\frac{1}{2} M \omega_{\text {eff }}^{2} r^{2}=\mu_{\text {eff }}^{m} \alpha_{m} X^{2}$ as well as the vortex azimuthal quantum kinetic energy $T_{v}=$ $m^{2} \hbar^{2} / 2 M r^{2}=m^{2} \mu_{\text {eff }}^{m} / X^{2}$. Therefore $\quad E_{\text {eff }}=\frac{1}{2} \times$ $\left(\mu_{\mathrm{eff}}^{m}+\left\langle H_{\mathrm{eff}}\right\rangle\right)$ leads to the ZTF condensate atom energy $E$ given by Eq. (5b).

Figure 1 shows in the thick dashed line the TF solution corresponding to the experimental setup described in Ref. [10] compared with the numerical solution of the GP differential Eq. (7) (solid continuous line) and the experimental data displayed by crosses (respectively, 


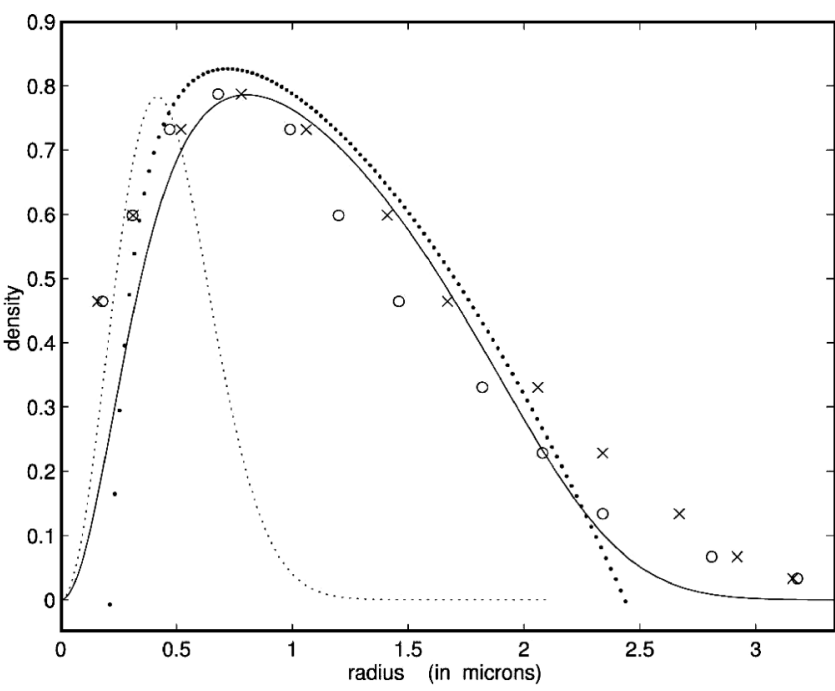

FIG. 1. The square amplitude $u_{1}^{2}$ of the $m=1$ single centered vortex defined by Eqs. (7) and (8) (solid continuous line), compared with the corresponding unnormalized (same amplitude) linear solution (thin dotted line), the semiclassical TF solution (thick dotted line), and the experimental data [Fig. 1b of Ref. [10]: the crosses (circles) display left (right) wing data].

circles) for the left (respectively, right) radial wing of the first centered vortex. The relevance to the TF approximation is quite convincing.

IV. ENS, JILA, and MIT axisymmetric vortex nucleation thresholds. - Let us now consider the basic $m=1$ single centered vortex case. Indeed the $m=2$ double-quantized vortex state is known to be unstable and splits into a pair of two $m=1$ vortices plus surface waves [7,27,28]. We also neglect any bending effect on the vortex line [29].

The present Letter suggests a vortex nucleation mechanism that is related to the resonance $\tilde{\Omega}=\left\langle\tilde{\Omega}_{1}(r)\right\rangle$ between the external rotation frequency $\tilde{\Omega}$ (in units of the transverse harmonic frequency $\omega_{\perp}$, hence the tilde overscript) and the diamagnetic ZTF quantum expectation value

$$
\left\langle\tilde{\Omega}_{1}(r)\right\rangle=\frac{2 \alpha_{1}-\ln \alpha_{1}-2}{2 \mathcal{N}} \sqrt{\frac{1+\tilde{\Omega}^{2}}{\alpha_{1}}}
$$

of the $L_{z}=\hbar$ vortex differential angular velocity

$$
\tilde{\Omega}_{1}(r)=\frac{\hbar}{M r^{2} \omega_{\perp}}=2 \frac{\mu_{\mathrm{eff}}^{1}}{\hbar \omega_{\perp}} \frac{1}{X^{2}}=\sqrt{\frac{1+\tilde{\Omega}^{2}}{\alpha_{1}}} \frac{1}{X^{2}}
$$

[cf. Eqs. (6)]. Hence the vortex nucleation threshold $\tilde{\Omega}_{v}=$ $\tilde{\Omega}_{0} / \sqrt{1-\tilde{\Omega}_{0}^{2}}$, where $\tilde{\Omega}_{0}=\left(2 \alpha_{1}-\ln \alpha_{1}-2\right) /\left(2 \mathcal{N} \sqrt{\alpha_{1}}\right)$.

(a) ENS' large laser-beam stirrer experiment. -The $N=1.4 \times 10^{5} \mathrm{Rb}^{87}$ condensate atoms are stirred by a large laser beam (whose waist is much larger than the radial condensate size) while being confined in an axially symmetric prolate (cigar shaped) harmonic potential whose average transverse and axial frequencies, respec- tively, are $\omega_{\perp} / 2 \pi=226 \mathrm{~Hz}$ and $\omega_{z} / 2 \pi=11.7 \mathrm{~Hz}$. The positive scattering length $a_{s} \sim 5.3 \times 10^{-7} \mathrm{~cm}$ for $\mathrm{Rb}^{87}$ $[30,31]$ and the full extend $\Delta_{z}=9.8 \times 10^{-3} \mathrm{~cm}$ of the BEC along the rotation axis yield $\mathcal{N}=30.29$ by use of Eq. (8) [32]. With these data, we have $\alpha_{1}=7.54 \times 10^{-3}$ by use of Eq. (9) and therefore $\tilde{\Omega}_{0}=0.552$ by use of Eq. (10). Hence the vortex resonance threshold $\tilde{\Omega}_{v}=$ $\Omega_{v} / \omega_{\perp}=\tilde{\Omega}_{0} / \sqrt{1-\tilde{\Omega}_{0}^{2}}=0.66$ which is in excellent agreement with the first centered vortex nucleation threshold $\tilde{\Omega}_{\exp }=0.65$ that has been obtained from the so-called procedure II stirring experimental setup, while the threshold related to the adiabatic stirring procedure I lies at $\tilde{\Omega}=$ $0.75[10-12,33]$.

(b) MIT's small laser-beam stirrer experiment.-The central observation of the MIT experiments $[13,14]$ states that a localized laser-beam stirrer (i.e., whose waist is smaller than the radial condensate size) can generate vortices at much lower stir frequencies than the minimum rotational frequency $\Omega_{s}=\min _{l}\left(\Omega_{l} / l\right)$ required to induce surface wave excitations with angular momentum $l \hbar$ and frequency $\Omega_{l}$ [16]. Indeed they found the first vortex nucleation threshold at the frequency $\Omega_{\exp } / 2 \pi=7 \mathrm{~Hz}$ while $\Omega_{s} / 2 \pi \geq 21 \mathrm{~Hz}$ for their experimental setup $[13,14,16]$. The authors emphasize that this paradoxical experimental result demonstrates that the surface mode analysis of vortex nucleation is incomplete since one might expect a localized stirrer to couple to many modes $l$. The present theory yields $\Omega_{v} / 2 \pi=9.5 \mathrm{~Hz}$ for the MIT experiments and thus provides a convincing argument in favor of our ZTF resonant-drive mechanism. Indeed, the MIT experiment consists in $N=5 \times 10^{7}$ condensate sodium atoms with a peak density equal to $4 \times 10^{14} \mathrm{~cm}^{-3}$. The healing length $0.2 \mu \mathrm{m}$ yields the positive scattering length $a_{s}=2.5 \times 10^{-7} \mathrm{~cm}$ while the condensate chemical potential $\mu=300 \mathrm{nK}$, together with the mean radial frequency $\omega_{\perp} / 2 \pi=86 \mathrm{~Hz}$, leads to $\mu / \hbar \omega_{\perp}=72.61$. Therefore we obtain $\alpha_{1}=\left(\hbar \omega_{\perp} / 2 \mu\right)^{2}=4.74 \times 10^{-5}$ in accordance with Eqs. (6). Hence $\tilde{\Omega}_{0}=0.11$, which yields the vortex resonance at $\Omega_{v} / 2 \pi=9.5 \mathrm{~Hz}$, to be compared with its experimental counterpart $\Omega_{\exp } / 2 \pi=7 \mathrm{~Hz}$.

(c) JILA's rotating normal-cloud experiment.-The JILA experiment consists in $N=5.5 \times 10^{5}$ condensate $\mathrm{Rb}^{87}$ atoms (scattering length $a_{s}=5.5 \times 10^{-7} \mathrm{~cm}$ ) confined in the static prolate harmonic potential $\left\{\omega_{\perp}, \omega_{z}\right\}=$ $2 \pi\{8.35,5.45\} \mathrm{Hz}$. The chemical potential (in units of $\left.\hbar \omega_{\perp}\right)$ is [31] $\mu=\frac{1}{2}\left[\left(15 N a_{s} \omega_{z}\right) /\left(\omega_{\perp} a_{\perp}\right)\right]^{2 / 5}=18.12$ since the transverse harmonic oscillator length $a_{\perp}=$ $\sqrt{\hbar /\left(M \omega_{\perp}\right)}=3.75 \times 10^{-4} \mathrm{~cm}$. Therefore $\alpha_{1}=$ $\left(\hbar \omega_{\perp} / 2 \mu\right)^{2}=7.61 \times 10^{-4}$ in accordance with Eqs. (6). Hence $\tilde{\Omega}_{0}=0.29$, which yields the vortex resonance at $\tilde{\Omega}_{v} / 2 \pi=0.30$, to be compared with the experimental interval $[0.32,0.38]$ where the first vortex is nucleated.

We can improve our predictions with respect to the JILA observations by proceeding in a slightly different way. Let 
us use [31] $\Delta_{z}=2\left[8 \pi N a_{s} a_{z}^{4}\right]^{1 / 5}=6.5 \times 10^{-3} \mathrm{~cm}$ since $a_{z}=\sqrt{\hbar /\left(M \omega_{z}\right)}=4.64 \times 10^{-4} \mathrm{~cm}$. Then $2 \mathcal{N}=$ $8 N a_{s} / \Delta_{z}=374.7$ in accordance with Eq. (8). Then Eq. (9) with $m=1$ yields $\alpha_{1}=1.31 \times 10^{-3}$ and therefore $\tilde{\Omega}_{0}=0.34$. Hence the vortex resonance threshold $\tilde{\Omega}_{v}=$ $\Omega_{v} / \omega_{\perp}=\tilde{\Omega}_{0} / \sqrt{1-\tilde{\Omega}_{0}^{2}}=0.36$, which is in excellent agreement with the first vortex nucleation interval $[0.32,0.38]$ that has been observed by the JILA group.

V. Conclusions. - The Zeeman Hamiltonian given by Eqs. (1) and (2) models the external rotation field $\overrightarrow{\boldsymbol{\Omega}}$ in which the dilute gaseous atomic BEC is embedded in quite the same sense as if it were embedded in the corresponding Larmor magnetic field. It yields in cylindrical symmetry both the $-\Omega L_{z}$ linear paramagnetic Zeeman coupling in the expression of the condensate energy $E$ per particle as well as its quadratic diamagnetic $\sqrt{1+\tilde{\Omega}^{2}}$ dependence in $\Omega$. The ZTF resonant-drive mechanism described in this Letter agrees with the results of the rotating BEC experiments using a laser-beam stirring process, with a large (ENS [10-12]) or a small localized (MIT [13,14]) beam waist. That it also explains the results of the JILA static potential experiment (which constitutes the opposite physically relevant situation, namely first rotating the normal cloud and then cooling it down below the condensation temperature [9]) is not the least argument in favor of the present model. Note, moreover, that, in agreement with observations, the present ZTF model does not necessarily imply first-order phase transition hysteresis behavior as predicted by other theories $[19,20]$. Let us finally stress that the role of discrete surface modes in BEC vortex formation still needs to be clarified: while the quadrupole dynamical instability seems indeed to be concomitant with both the ENS [17] and the 3-point pattern MIT [13] largebeam stirrer experiments, it does not occur in those MIT vortex nucleation experiments that use a small beam waist stirrer nor in the JILA rotating normal cloud ones [9].

The author acknowledges the referees for their helpful criticisms concerning previous-as well as presentattempts in this research. The author is deeply grateful to Y. Castin, E. A. Cornell, J. Dalibard, G. Kavoulakis, C. J. Pethick, P. Valiron, and Yongle Yu for several very useful comments. The author also acknowledges the hospitality of the Science Institute of the University of Iceland (Reykjavik) and warmly thanks G. Björnsson and V. Gudmundsson for numerous fruitful discussions there.

[1] M. H. Anderson et al., Science 269, 198 (1995).

[2] C. C. Bradley, C. A. Sackett, J. J. Tollet, and R. G. Hulet, Phys. Rev. Lett. 75, 1687 (1995).

[3] K. B. Davis et al., Phys. Rev. Lett. 75, 3969 (1995).
[4] F. Dalfovo, S. Giorgini, L. P. Pitaevskii, and S. Stringari, Rev. Mod. Phys. 71, 463 (1999).

[5] A. J. Leggett, Rev. Mod. Phys. 73, 307 (2001).

[6] R. J. Donnelly, Quantized Vortices in Helium II (Cambridge University Press, Cambridge, 1991).

[7] D. A. Butts and D. S. Rokhsar, Nature (London) 397, 327 (1999).

[8] M. R. Matthews, B. P. Anderson, P. C. Haljan, D. S. Hall, C. E. Wieman, and E. A. Cornell, Phys. Rev. Lett. 83, 2498 (1999).

[9] P. C. Haljan, I. Coddington, P. Engels, and E. A. Cornell, Phys. Rev. Lett. 87, 210403 (2001).

[10] F. W. Madison, C.F. Chevy, W. Wohlleben, and J. Dalibard, Phys. Rev. Lett. 84, 806 (2000).

[11] C. F. Chevy, F. W. Madison, and J. Dalibard, Phys. Rev. Lett. 85, 2223 (2000).

[12] F. W. Madison, C. F. Chevy, V. Bretin, and J. Dalibard, Phys. Rev. Lett. 86, 4443 (2001).

[13] C. Raman, J. R. Abo-Shaeer, J. M. Vogels, K. Xu, and W. Ketterle, Phys. Rev. Lett. 87, 210402 (2001).

[14] J.R. Abo-Shaeer, C. Raman, J.M. Vogels, and W. Ketterle, Science 292, 476 (2001).

[15] C. Cohen-Tannoudji, J. Dalibard, and F. Laloe, Einstein Aujourd'hui (EDP Sciences, Paris, 2005).

[16] F. Dalfovo and S. Stringari, Phys. Rev. A 63, 011601(R) (2000).

[17] S. Sinha and Y. Castin, Phys. Rev. Lett. 87, 190402 (2001).

[18] C. Lobo, A. Sinatra, and Y. Castin, Phys. Rev. Lett. 92, 020403 (2004).

[19] J. J. Garcia-Ripoll and V. M. Perez-Garcia, Phys. Rev. A 63, 041603 (2001).

[20] G. Kavoulakis, Eur. Phys. J. D 36, 11 (2005).

[21] F. Dalfovo and S. Stringari, Phys. Rev. A 53, 2477 (1996).

[22] T.-L. Ho, Phys. Rev. Lett. 87, 060403 (2001).

[23] N. R. Cooper, N. K. Wilkin, and J. M. F. Gunn, Phys. Rev. Lett. 87, 120405 (2001).

[24] S. Stock, B. Battelier, V. Bretin, Z. Hadzibabic, and J. Dalibard, Laser Phys. Lett. 2, 275 (2005).

[25] A. Aftalion, X. Blanc, and J. Dalibard, Phys. Rev. A 71, 023611 (2005).

[26] C. J. Pethick and H. Smith, Bose-Einstein Condensations in Dilute Gases (Cambridge University Press, Cambridge, 2004).

[27] J. J. Garcia-Ripoll and V. M. Perez-Garcia, Phys. Rev. A 60, 4864 (1999).

[28] G. M. Kavoulakis, B. Mottelson, and C. J. Pethick, Phys. Rev. A 62, 063605 (2000).

[29] M. Modugno, L. Pricoupenko, and Y. Castin, Eur. Phys. J. D 22, 235 (2003).

[30] D. L. Feder, C. W. Clark, and B. I. Schneider, Phys. Rev. Lett. 82, 4956 (1999).

[31] G. Baym and C. J. Pethick, Phys. Rev. Lett. 76, 6 (1996).

[32] The nonlinearity parameter $U=4 \pi N a_{S} / a_{\perp}$ that is used in Ref. [19] equals 13000 in the ENS experiment Ref. [10] while the results displayed by Fig. 1 of Ref. [19] assume $0 \leq U \leq 4000$; therefore, they cannot concern the ENS experiment.

[33] S. Sinha and Y. Castin, Phys. Rev. Lett. 87, 190402 (2001). 